\title{
Thermal correction of the radii of curvature of mirrors for GEO 600
}

\author{
H Lück ${ }^{1}$, A Freise ${ }^{2}$, S Goßler ${ }^{1}$, S Hild ${ }^{1}$, K Kawabe ${ }^{1}$ and K Danzmann ${ }^{1}$ \\ ${ }^{1}$ Max-Planck-Institute for Gravitational Physics (Albert-Einstein-Institute) and University of \\ Hannover, Callinstr. 38, D-30167 Hannover, Germany \\ 2 European Gravitational Observatory, Via E. Amaldi, 56021 Cascina (PI), Italy \\ E-mail: hal@aei.mpg.de
}

Received 29 August 2003

Published 11 February 2004

Online at stacks.iop.org/CQG/21/S985 (DOI: 10.1088/0264-9381/21/5/090)

\begin{abstract}
A mismatch of the radii of curvature of the mirrors in the arms of an interferometric gravitational-wave detector can be partly compensated by creating a thermal gradient inside the mirror. This paper shows how the interference quality at the output of the German/British GEO 600 gravitationalwave detector could be improved with a simple ring heater.
\end{abstract}

PACS number: $04.80 . \mathrm{Nn}$

(Some figures in this article are in colour only in the electronic version)

\section{Introduction}

High power recycling factors of up to 2000 in the German/British gravitational-wave detector GEO 600 [1] require an excellent cancellation of the two beams returning from the interferometer arms at the beam splitter in order to obtain low enough losses through the output port.

GEO 600 is a dual recycled Michelson interferometer with folded arms (see figure 1) and an arm length of $600 \mathrm{~m}$. The power recycling mirror currently has a transmission of $1.35 \%$ which allows the power recycling factor to reach about $300(=4 / 1.35 \%)$. In the final configuration a power recycling mirror with a transmission of only $0.1 \%$ will be used.

For an optically stable operation of the power- and signal-recycling cavities the far folding mirrors were designed to have a radius of curvature (ROC) of $640 \mathrm{~m} \mathrm{[3].} \mathrm{After} \mathrm{coating,} \mathrm{the}$ actual ROC of the optics turned out to be $666 \mathrm{~m}$ and $687 \mathrm{~m}$ for the north and east folding mirrors, respectively.

Thus, the wavefronts returning from the two arms had different ROC at the beam splitter and could not interfere well enough. The resulting losses at the output port of about $0.25 \%$ prevented the power build-up in the power recycling cavity from becoming more than 200 . The resulting 'sombrero'-like beam shape can be seen in figure 6 . 


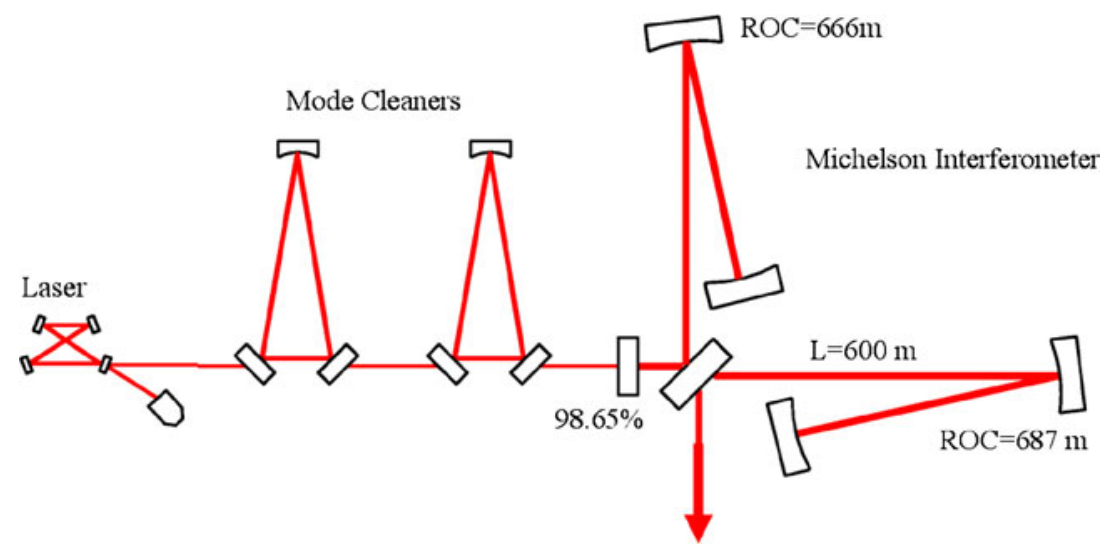

Figure 1. Schematics of the optical layout of GEO 600. A signal recycling mirror in the output was installed during the measurements of this paper but was not used.

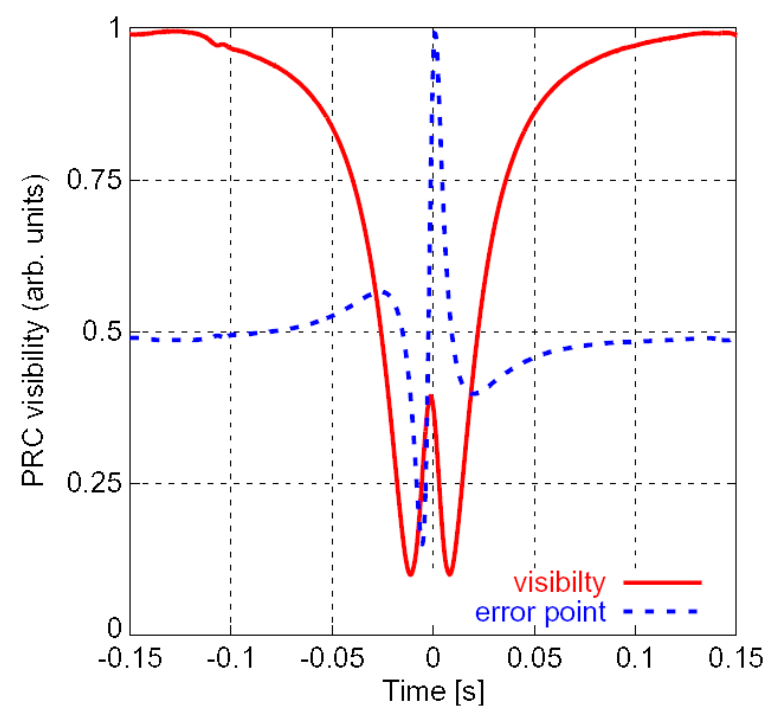

Figure 2. Measured differential Michelson error point signal and reflected power at the input port with good interferometer contrast.

In addition to the lowered power build-up the longitudinal control signal is unfavourably affected by the ROC mismatch. Figure 2 shows the error point signal for the longitudinal differential Michelson control together with the reflected power at the input port for a moderately good interference contrast while the arm length difference passes the operating point (dark fringe). The error point shows a steep slope at the dark fringe and about $40 \%$ of the input power is reflected as the power recycling cavity becomes strongly overcoupled. If the interferometer gets misaligned or if the returning beams do not cancel well due to a mismatch in the ROC (figure 3) then the error point signal becomes smaller and hence the slope at the operating points reduces until it completely vanishes when the losses match the transmittance of the power recycling mirror and the power recycling cavity is impedance matched. If the 


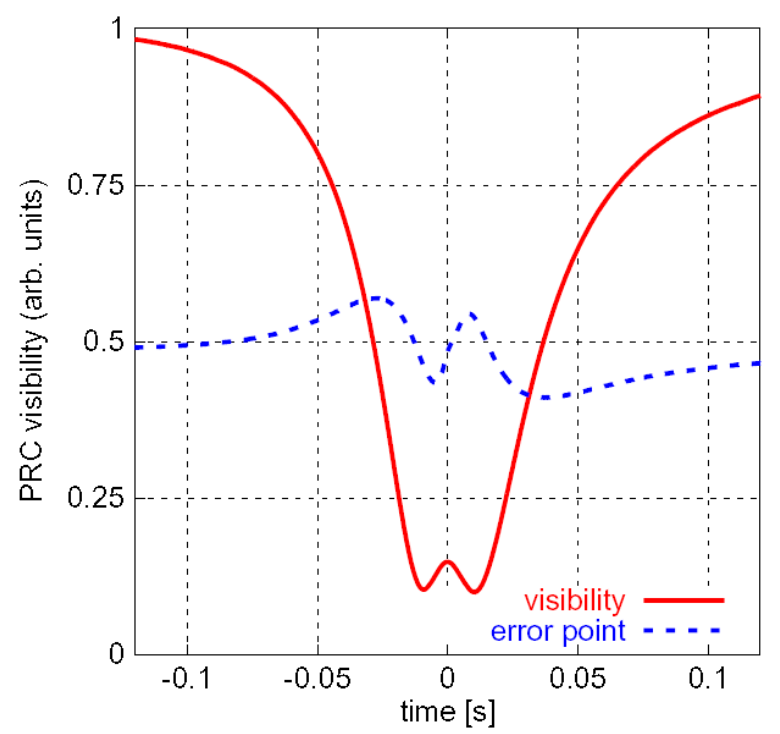

Figure 3. Measured differential Michelson error point signal and reflected power at the input port with poor interferometer contrast.

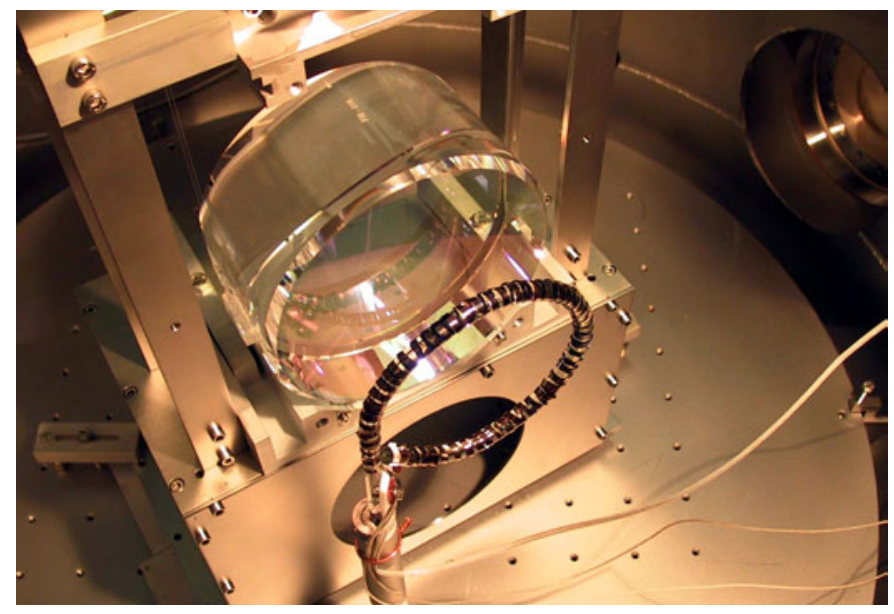

Figure 4. The heater behind the east folding mirror.

losses due to the ROC mismatch are already big, small misalignments will cause the control signal to become so small that the interferometer loses lock.

\section{Thermal adjustment of the mirror radius of curvature}

The GEO 600 mirrors are made of fused silica (Suprasil 1 from Heraeus) with a diameter of $18 \mathrm{~cm}$ and a thickness of $10 \mathrm{~cm}$. We adjust the ROC of the mirrors with radiative heating from a ring heater located about $5 \mathrm{~cm}$ behind the east far folding mirror (see figure 4). The heater is made of a ring-like Duran glass rod wrapped with a $100 \mu \mathrm{m}$ thick stainless steel ribbon which is heated by passing a DC current through it. Before inserting it into the GEO 600 


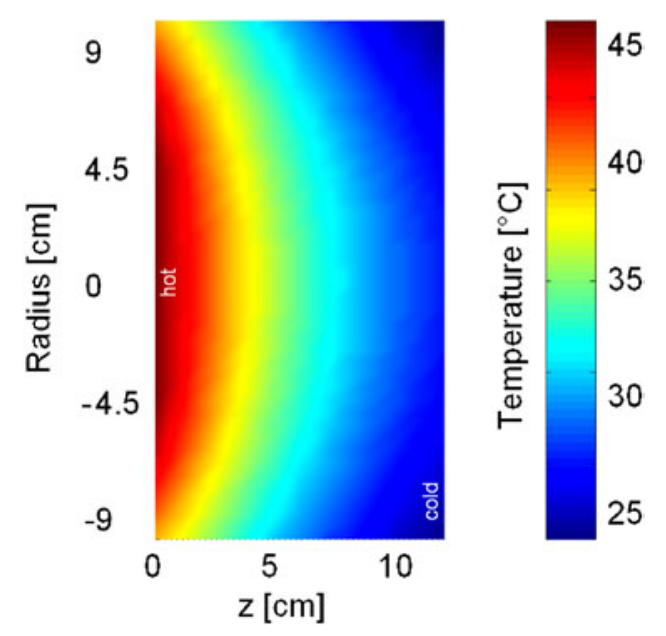

Figure 5. Analytical calculation of the temperature distribution inside the mirror substrate for a heater power of about $50 \mathrm{~W}$. The heater is placed on the left side of the mirror. The mirror surface is at the right. The temperature corresponding to the various grey levels is indicated in the right bar.
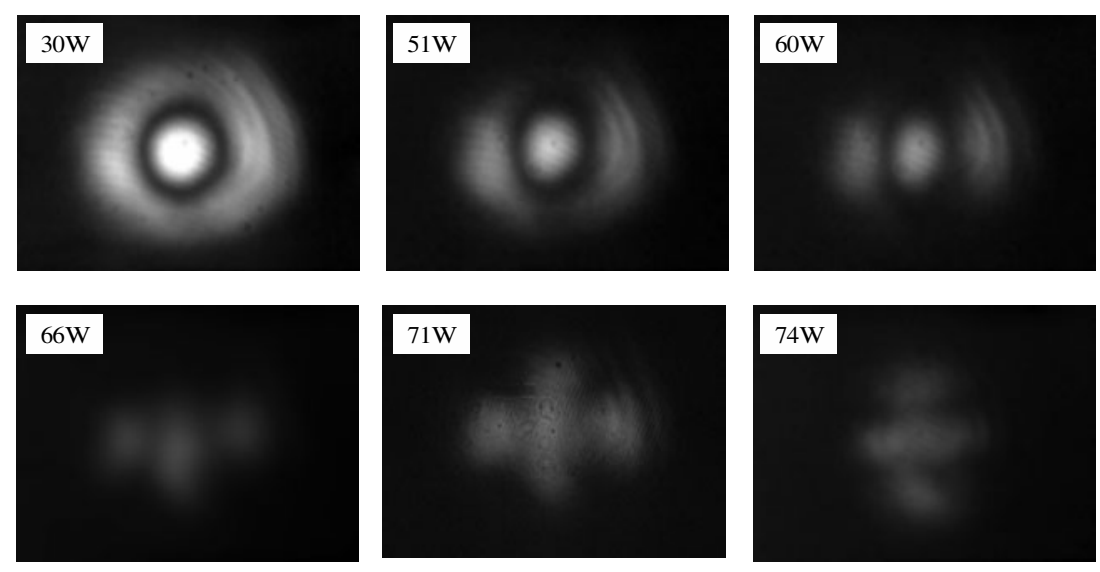

Figure 6. Interference pattern at the output port of the interferometer for different heater powers (indicated). In the lower row the brightness is slightly enhanced for better visibility.

vacuum system it was given an air bake and a vacuum bake after cleaning. The heater creates a thermal gradient inside the mirror (see figure 5) and thermal expansion decreases the ROC of the front (mirror) surface. By changing the heater power the ROC can be adjusted to the required value, i.e. $666 \mathrm{~m}$.

The effect on the output mode is shown in figure 6 . At a power of $66 \mathrm{~W}$ the mismatch in the ROCs is compensated in the vertical direction and only astigmatic effects remain. Astigmatism can originate from lateral thermal gradients in the mirror caused by a radiationwise asymmetrical environment, e.g. the catcher structure surrounding the mirror or by a slightly curved beam splitter surface ( $5 \mathrm{~km}$ ROC could explain this effect), which introduces astigmatism due to the angle of incidence of $43^{\circ}$. At $74 \mathrm{~W}$ the compensation is perfect for the horizontal direction and the pattern is rotated by $90^{\circ} .71 \mathrm{~W}$ is the optimum in terms 


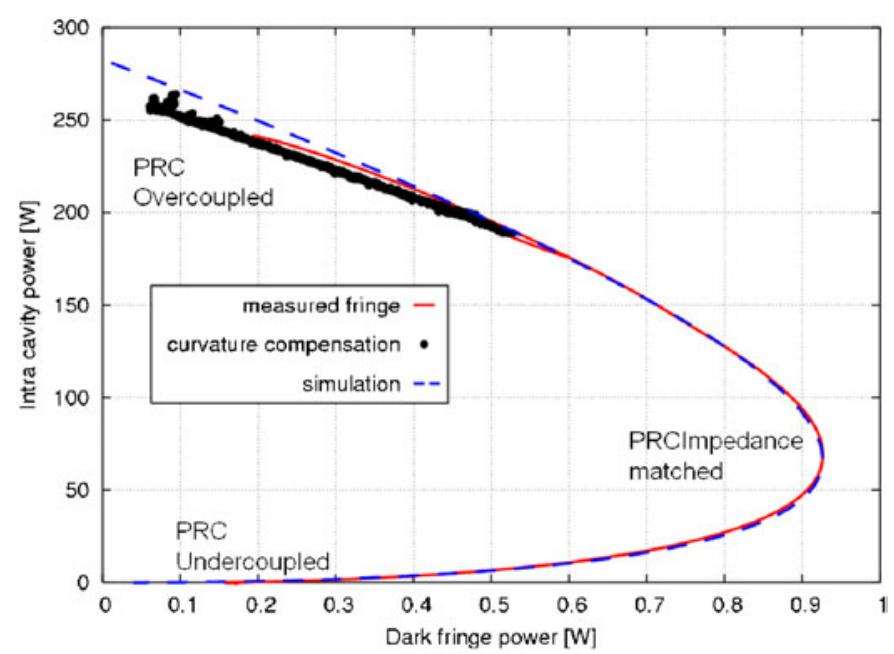

Figure 7. The power inside the power recycling cavity (PRC) as a function of the power at the output port. The dashed line shows a simulation with the software FINESSE [2] whereas the solid line shows a measurement when the interferometer passes the dark fringe. The dots show the dependence for different heater powers.

of dark port power. The astigmatism can be compensated by using a segmented heater and thus introducing asymmetric heating and deformation. So far this was not needed for our application. The increase in the intracavity power and the improvement in the dark port power during the ROC compensation can be seen in figure 7. Varying the heater power from $0 \mathrm{~W}$ to $65 \mathrm{~W}$ improved the contrast at the dark port by an order of magnitude. A further increase of the heater power to $71 \mathrm{~W}$ (not shown in figure 7) decreased the dark port power to about $20 \mathrm{~mW}$ at an intracavity power close to $300 \mathrm{~W}$.

\section{References}

[1] Willke B et al 2004 Status of the GEO 600 detector Class. Quantum Grav. 21 S417

[2] Freise A et al 2004 Frequency domain interferometer simulation with higher-order spatial modes Class. Quantum Grav. $21 \mathrm{~S} 1067$

[3] Winkler W 2004 to be published 